\title{
Short communication: Colostrum versus formula: Effects on mRNA expression of genes related to branched-chain amino acid metabolism in neonatal dairy calves
}

\author{
Morteza H. Ghaffari, ${ }^{1}$ Hassan Sadri, ${ }^{2 *} †$ Harald M. Hammon, ${ }^{3}$ Julia Steinhoff-Wagner, ${ }^{3} \ddagger$ Nico Henschel, ${ }^{1}$ \\ and Helga Sauerwein ${ }^{1}$ \\ ${ }^{1}$ Institute of Animal Science, Physiology Unit, University of Bonn, 53111 Bonn, Germany \\ ${ }^{2}$ Department of Clinical Science, Faculty of Veterinary Medicine, University of Tabriz, 5166616471 Tabriz, Iran \\ ${ }^{3}$ Institute of Nutritional Physiology, Oskar Kellner, Leibniz Institute for Farm Animal Biology, 18196 Dummerstorf, Germany
}

\begin{abstract}
The objective of the current study was to elucidate the effect of feeding colostrum or milk-based formula on the tissue mRNA abundance of the most relevant branched-chain amino acids (BCAA) transporters and catabolizing enzymes in newborn calves. German Holstein calves were fed either colostrum (COL; $\mathrm{n}=7$ ) or milk-based formula (FOR; $\mathrm{n}=7$ ) with comparable nutrient composition but lower contents of free BCAA, insulin, and insulin-like growth factor-I in the formula than in the respective colostrum for up to $4 \mathrm{~d}$ of life. Tissue samples from liver, kidney fat, 3 different muscles [M. longissimus dorsi (MLD), M. semitendinosus (MST), and M. masseter (MM)], as well as duodenum, jejunum, and ileum were collected following euthanasia on $\mathrm{d} 4$ at $2 \mathrm{~h}$ after feeding. The plasma-free BCAA were analyzed, and the tissue abundance of solute carrier family 1 member 5 (SLC1A5), SLC7A5, and SLC38A2 as well as mitochondrial isoform of branched-chain aminotransferase (BCATm), branched-chain $\alpha$-keto acid dehydrogenase $\mathrm{E} 1 \alpha(B C K D H A)$, and branchedchain $\alpha$-keto acid dehydrogenase $\operatorname{E} 1 \beta(B C K D H B)$ were assessed. The preprandial plasma concentrations of free BCAA were affected by time but did not differ between groups. The plasma concentrations of free BCAA decreased in COL, whereas they increased in FOR after feeding, resulting in higher postprandial plasma total BCAA concentrations in FOR than in COL. The mRNA abundances of BCATm, BCKDHA, $B C K D H B$, as well as BCAA transporters in the liver,
\end{abstract}

Received February 26, 2020.

Accepted June 4, 2020.

*Corresponding author: sadri@tabrizu.ac.ir

$\dagger$ H. Sadri was a visiting scientist at the Institute of Animal Science, Physiology Unit, University of Bonn, 53115 Bonn, Germany at the time the research was done.

$\ddagger$ Present address: Institute of Animal Science, Preventive Health Unit, University of Bonn, 53111 Bonn, Germany. were not affected by the diet. In kidney fat, the mRNA abundance of BCAA catabolizing enzymes did not differ between groups, but that of $S L C 1 A 5$ was lower in FOR than in COL. The mRNA abundance of BCAA catabolizing enzymes in different sections of the small intestine was not affected by the diet, whereas that of $S L C^{r} 7 A 5$ was or tended to be lower in the duodenum, proximal jejunum, and mid jejunum of the COL calves compared with the FOR calves. The mRNA abundance of $B C K D H A$ was lower in MLD and MM but greater in MS for the FOR calves compared with the COL calves. The mRNA abundance of SLC7A5 in MST was lower in FOR than in COL, whereas it was unaffected by the diet in MLD and MM. The differential effect of feeding colostrum on the mRNA abundance of BCKDHA in 3 different muscle tissues might point to a muscle typespecific response. The results also indicate that the colostral BCAA might be favorably used for anabolic metabolism in the small intestine of neonatal calves. Such effects are speculated to be due to the stimulatory effects of growth factors and hormones present in colostrum

Key words: colostrum, branched-chain amino acid transporter, branched-chain amino acid enzyme, neonatal calf

\section{Short Communication}

Neonatal calves need to adapt to extrauterine life and have to cope with energy intake shifting from a continuous maternal supply of nutrients via the placenta toward discontinuous supply via colostrum and milk or milk replacer intake (Hammon et al., 2012). Besides the supply of immunoglobulins, feeding colostrum to neonatal calves has several positive effects, such as stimulating morphological growth and functional maturation of the gastrointestinal (GI) tract (Bühler et al., 1998; Blum and Hammon, 2000; Hernández-Castellano et al., 2015), promoting the colonization with beneficial 
bacteria (Malmuthuge et al., 2015; Ma et al., 2019), improving glucose status by enhanced intestinal glucose absorption due to the advanced development of the intestinal epithelia (Steinhoff-Wagner et al., 2011; Steinhoff-Wagner et al., 2014; Gruse et al., 2015), and reducing the susceptibility to diarrhea and pneumonia (Kargar et al., 2020). The colostrum feeding also affects the circulating amino acid concentrations, as previously shown for neonatal calves (Hammon and Blum, 1999; Sadri et al., 2017).

The branched-chain amino acids (BCAA), namely leucine, isoleucine, and valine, are part of a group of essential AA that play an important role in neonatal nutrition (de Groof et al., 2014; Kashyap and Heird, 2011; Suryawan and Davis, 2011), and in various physiological and metabolic functions (Zhang et al., 2017). The metabolism of BCAA is a complex process that begins with the reversible deamination catalyzed by the branched-chain aminotransferase (BCAT; EC 2.6.1.42), yielding the respective branched-chain $\alpha$-keto acids (BCKA; ketoisoleucine, ketoleucine, and ketovaline) and glutamate and occurring primarily in extrahepatic tissues including muscle and adipose tissue (Neinast et al., 2019). Subsequently, the irreversible oxidative decarboxylation of the BCKA to branched-chain acyl CoA derivatives is catalyzed by the rate-limiting branched-chain $\alpha$-keto acid dehydrogenase (BCKDH; EC 1.2.4.4) complex (Neinast et al., 2019) that might act in the liver again to maintain physiological BCAA and BCKA levels (Ananieva et al., 2017; Webb et al., 2019). As BCAA can be potentially transaminated in several tissues, whole-body BCAA homeostasis is thus highly dependent on the crosstalk between various tissues (David et al., 2019).

Amino acid transporters are transmembrane proteins that mediate the transfer of AA into and out of cells; they are also important in regulating energy metabolism, protein synthesis, and gene expression at the cellular and whole-body level (Kandasamy et al., 2018). In case of BCAA, the preferred transporter is the sodium-independent L-type large neutral amino acid transporter small subunit 1 (LAT1; encoded by the solute carrier family 7-member 5 ( $S L C 7 A 5$ gene), which imports Leu and other BCAA into cells in exchange for Gln (Hyde et al., 2003; Batistel et al., 2017). Other neutral transporters such as the A-type sodium-coupled neutral amino acid transporter 2 (SNAT2, encoded by SLC38A2) and the ASC-type sodium-dependent neutral amino acid transporter type 2 (ASCT2, encoded by $S L C 1 A 5$ ) also mediate BCAA transport through net Gln uptake both directly and indirectly (Hundal and Taylor, 2009). So far, the tissue abundance of BCAA transporters has been assessed in dairy cows (Bionaz and Loor, 2011; Batistel et al., 2017; Liang et al., 2019;
Webb et al., 2020); no studies have been conducted in dairy calves. A simplified schematic representation of the BCAA uptake by the LAT1, SNAT2, and ASCT2 as well as the catabolism of the BCAA pathway is depicted in Supplemental Figure S1 (https://doi.org/10 $.3168 /$ jds.2020-18429).

In the current study, we hypothesized that the expression of BCAA catabolic enzymes and their transporters in different tissues of neonatal calves is divergently affected by colostrum versus milk-based formula (with lower contents of free BCAA, insulin, and IGF-I than in colostrum) fed for up to $4 \mathrm{~d}$ of life. To test this hypothesis, we used neonatal calves receiving either colostrum or formula and investigated the differences in the mRNA abundance of the most relevant BCAA transporters as well as the mRNA abundance of the BCAA catabolizing enzymes in hepatic and extrahepatic tissues at $\mathrm{d} 4$ of life.

This experiment was conducted at the Research Station of the University of Rostock (Rostock, Germany) from September 2007 until March 2008. The regulations of the German Animal Welfare Act (TierSchG) in its respective current edition were met. All animal experiments were approved by the relevant authorities of the State Mecklenburg-Vorpommern, Germany (LALLFM-V/TSD/7221.3-1.1-014/07). Fourteen male German Holstein-Friesian calves were immediately separated from their dams after birth and randomly assigned by birth weight to 2 treatment groups (Supplemental Figure S1, https://doi.org/10.3168/jds .2020-18429). Calves were fed either colostrum (COL; $\mathrm{n}=7$ ) or milk-based formula (FOR; $\mathrm{n}=7)$. The COL calves were fed pooled colostrum of milkings 1,3 , and 5 (d 1, 2, and 3 after calving, respectively) during their first $3 \mathrm{~d}$ of life, followed by pooled transition milk of milking from d 3 on d 4 . The FOR calves were fed 3 differently composed milk-based formulas on $\mathrm{d} 1,2$, and 3 , followed by formula from d 3 on $\mathrm{d} 4$. The formula contained comparable amounts of macronutrients as the respective colostrum milkings (Supplemental Table S1, https://doi.org/10.3168/jds.2020-18429; SteinhoffWagner et al., 2011). Colostrum and formula-fed amounts were targeted to be $8 \%$ of BW on d 1 and $10 \%$ of BW from d 2 on. Calves were bottle-fed twice daily from d 1 to 3 (0800 and $1600 \mathrm{~h}$ ) and once $(0800 \mathrm{~h}$ ) on d 4. Colostrum and transition milk were obtained from milkings 1, 3, and 5 after calving from cows of the farm where calves were born. Individual pools of colostrum milkings were prepared before the study started, deepfrozen in portions, and heated to $37^{\circ} \mathrm{C}$ by water bath before feeding. Calves in the FOR group were fed with a milk-based formula (Bergophor Futtermittelfabrik GmbH, Kulmbach, Germany) specifically blended for this study with comparable nutrient contents to those 
of the respective colostrum especially regarding lactose content, but lacking substantial amounts of biologically active factors such as IGF-I or insulin, as described earlier (Supplemental Table S1; Steinhoff-Wagner et al., 2011). The formula was mixed as a bulk, analyzed, deep-frozen in portions, and heated to $37^{\circ} \mathrm{C}$ by water bath before feeding. Moreover, to ensure comparable lactose contents in colostrum and related formulas, 77, 39 , and $42 \mathrm{~g}$ lactose $/ \mathrm{kg}$ of $\mathrm{DM}$ were added to colostrum milkings 1,3 , and 5 , respectively. To ensure that all calves received an equal amount of formula or colostrum, calves with reduced appetite were tube-fed their residual amount of milk. Tube feeding was necessary for $7 \mathrm{FOR}$ and $3 \mathrm{COL}$ calves once on $\mathrm{d} 1$ to $\mathrm{d} 3$, but not on d 4 . On d 4, calves were fed either colostrum of milking 5 or formula of d 3 for groups COL and FOR, respectively. Samples of individual pools were collected during pooling and chemical composition was analyzed by the Qualitätsprüfungs- und Dienstleistungsgesellschaft Mecklenburg-Vorpommern mbH. Dry matter, $\mathrm{CP}$, crude fat, and ash were determined according to the Weender standard procedure (Naumann and Bassler, 2012). Insulin and IGF-I concentrations in colostrum and formula pool samples were measured by RIA as described in the footnotes of Supplemental Table S1.

The calves received their first meal $(8 \%$ of $\mathrm{BW}$ on $\mathrm{d}$ 1 and $10 \%$ of BW from d 2 onwards) $4.4 \pm 0.6 \mathrm{~h}$ after birth. All calves were singletons and spontaneously born to multiparous cows (lactation number 2 or 3 ) after normal gestation length. Calves were kept individually in indoor pens with straw bedding at the Research Station of the University of Rostock and had free access to water. On d 1, 2, and 3, calves were subcutaneously injected with antibiotics (25 mg enrofloxacin/10 kg of BW; Baytril 5\%; Bayer AG, Leverkusen, Germany) to prevent the risk of infection following catheterization. To avoid iron deficiency, calves received intramuscular injections of iron dextran $(10 \mathrm{mg} / \mathrm{kg}$ of BW; Ursoferran 100, Serumwerk Bernburg) before first feed intake. The health status was evaluated daily based on rectal temperature, heart rate, respiratory rate, behavior, nasal discharge, respiratory sounds, fecal consistency, and navel inspection. The health status, handling, and birth weight of COL and FOR calves were published previously (Steinhoff-Wagner et al., 2011).

All calves were stunned by captive bolt and exsanguinated on $\mathrm{d} 4$ of life, $2 \mathrm{~h}$ after the feeding. The abdominal cavity was opened, and the liver, 3 different muscles [M. longissimus dorsi (MLD), M. semitendinosus (MST), and M. masseter (MM)], kidney fat, and intestinal mucosa (duodenum, jejunum, and ileum) were immediately sampled. Tissue samples were snap frozen in liquid nitrogen and stored at $-80^{\circ} \mathrm{C}$ until analyzed.
Tissue samples were homogenized using the Precellys 24 system (peQLab Biotechnology, Erlangen, Germany). Total RNA was extracted from the homogenates using Qiazol (Qiagen, Hilden, Germany) and purified using spin columns (InviTrap Spin Universal RNA Mini Kit, stratec molecular, Birkenfeld, Germany). The concentration of RNA and the purity were controlled by absorbance readings at $260 \mathrm{~nm}$ and $280 \mathrm{~nm}$ using the Nanodrop 1000 (peQLab Biotechnology). The integrity of the RNA was assessed using ethidium bromide denaturing RNA gel electrophoresis. For cDNA synthesis, reverse transcription of $250 \mathrm{ng}$ of total RNA $/ 20 \mu \mathrm{L}$ of reaction volume was performed using $250 \mathrm{pmol}$ of random hexamer primers (Sigma-Aldrich, Nümbrecht, Germany) for $5 \mathrm{~min}$ at $65^{\circ} \mathrm{C}$ first, then adding $200 \mathrm{U}$ of Revert Aid reverse transcriptase, $10 \mathrm{mM}$ of each dNTP, and $20 \mathrm{U}$ of RiboLock ribonuclease inhibitor (Fermentas, St. Leon-Rot, Germany) and incubating for $10 \mathrm{~min}$ at $27^{\circ} \mathrm{C}, 60 \mathrm{~min}$ at $42^{\circ} \mathrm{C}$, and $1 \mathrm{~min}$ at $99^{\circ} \mathrm{C}$. Each run comprised a negative template control and a no-reverse transcriptase control. To reduce the variation in cDNA synthesis, reverse transcription was performed in duplicates from each tissue, which were then combined for quantitative PCR.

Analysis by quantitative reverse-transcription realtime PCR was performed in accordance with the minimum information for publication of quantitative realtime PCR experiments guidelines (Bustin et al., 2009). The primer sequences, as well as the real-time PCR conditions, are given in Supplemental Table S2 (https: //doi.org/10.3168/jds.2020-18429). Triplicates with a total volume of $10 \mu \mathrm{L}$ consisting of $2 \mu \mathrm{L}$ of $1: 4$ diluted cDNA as template, $5 \mu \mathrm{L}$ SYBR Green JumpStart Taq Readymix (Sigma-Aldrich, Steinheim, Germany), respectively, for BCKDHA and BCATm, Blue S'Green qPCR 2x mix (Biozym, Hessisch Oldendorf, Germany) or DyNAmo Flash SYBR Green qPCR Mix 2x (Thermo Scientific, Waltham, USA), $1 \mu \mathrm{L}$ of the assay-specific primer mix, and $2 \mu \mathrm{L}$ of water were run in an Mx3000P cycler (Agilent, Stratagene, Amsterdam, the Netherlands). All reactions contained a negative template control for $\mathrm{qPCR}$ and a negative template control and no-reverse transcriptase control of cDNA, as well as 2 diluted pools of the specific tissues cDNA as inter-run calibrator (IRCs, 1:4 and 1:8). For each PCR reaction, a standard curve was generated using a serial dilution of cDNA to calculate efficiency-corrected relative quantities of the targets. The software $\mathrm{qBASE}^{\text {plus }}$ version 3.2 (Biogazelle, Ghent, Belgium) was used to determine the most stably expressed reference genes for $\mathrm{qPCR}$ data normalization. Reference genes were evaluated based on their average expression stability $(\mathrm{M})$ and pairwise variation $(\mathrm{V})$ values (accepted $\mathrm{M}<1.5$ and $\mathrm{V}<0.15$ ) using the geNorm ${ }^{\text {PLUS }}$ algorithms of $q$ Base ${ }^{\text {PLUS }}$ software. 
The most stable reference genes for each tissue were determined as follows: different sections of the small intestine: glyceraldehyde-phosphate-dehydrogenase $(G A P D H)$, eucariotic translation initiation factor 3 (EIF3K); for the muscle tissues: EIF3K and ribosomal protein S9 (RPS9); for liver tissue: lipoprotein receptor-related protein 10 (LRP10) and RNA polymerase II (POLR2A); and for kidney fat: emerin $(E M D)$ and LRP10. All subsequent calculations and data quality controls were performed using qBASE ${ }^{\text {plus }}$ software (Hellemans et al., 2007). The output data from the software were calibrated normalized relative quantities.

The final results calculated by $\mathrm{qBASE}^{\text {plus }}$ (i.e., the calibrated normalized relative quantities values) were used for statistical analysis of the mRNA data. The data were statistically analyzed with estimation methods using Mann-Whitney U statistic (Crichton, 2000) and presented as mean difference estimation plots (Claridge-Chang and Assam, 2016). Effect size was measured using Hedges' $g$ (Greenland et al., 2016) as described elsewhere (Ghaffari et al., 2019; Sadri et al., $2020)$ and were referred to as trivial $(g<0.2)$, small $(0.2<g<0.5)$, moderate $(0.5<g<0.8)$, or large $(g$ $>0.8)$, as per standard practice.

The mRNA abundance of the enzymes related to BCAA catabolism and the $3 \mathrm{BCAA}$ transporters in the liver was not affected by the diet (Figure 1). The mRNA abundances of $B C A T m, B C K D H A, B C K D H B$, and 2 BCAA transporters (SLC7A5 and SLC38A2) were also not different between the groups in kidney fat (Figure 1). The mRNA abundance of SLC1A5 in kidney fat was substantially lower $(\mathrm{g}>-1 ; P<0.05)$ in FOR calves than in COL calves (Figure 1).

The mRNA abundances of BCATm, BCKDHA, $B C K D H B$, and 2 BCAA transporters (SLC1A5 and SLC38A2) were not different between groups in the duodenum, proximal jejunum, mid jejunum, distal jejunum, and ileum (Figure 2). The mRNA abundance of $S L C 7 A 5$ was greater $(P<0.05)$ with a high effect size in the duodenum and proximal jejunum, or tended to be greater in the mid jejunum $(P=0.09)$ in FOR calves than COL calves (Figure 2). However, the mRNA abundance of $S L C 7 A 5$ was similar between the groups in the distal jejunum and ileum (Figure 2).

The mRNA abundance of $B C K D H A$ was lower $(P$ $<0.05)$ with a high effect size in the MLD and MM, but was greater with a high effect size in the MST $(P$ $<0.05)$ for the FOR calves compared with the COL calves (Figure 3). The mRNA abundance of SLC7A5 was lower with a high effect size in the MST $(P<0.05)$ in FOR than in COL, whereas its mRNA abundance was unaffected by the diet in the MLD and MM (Figure $3)$. The mRNA abundances of $B C A T m$ and $B C K D H B$ and 2 BCAA transporters (SLC1A5 and SLC38A2) were not different between the groups in the 3 different skeletal muscles (Figure 3); except for the MM in which the SLC1A5 mRNA abundance tended $(P=0.09)$ to be lower in the FOR calves as compared with the COL calves.

As reported previously from this experiment (Sadri et al., 2017), colostrum had greater concentrations of free BCAA and free total AA than formula; however, plasma-free total AA and BCAA concentrations decreased in COL, whereas they increased in FOR after the feeding, resulting in higher postprandial BCAA concentrations in FOR than in COL. The first-pass metabolism is a complex process involving the gut and liver and can dictate the extent to which a nutrient reaches the circulation. Most essential AA are catabolized in the liver, whereas BCAA are removed by the liver (first-pass hepatic catabolism) to a lesser extent than most other AA due to the absence or very low activity of hepatic BCAT (Harper et al., 1984). The small intestine might thus play an important role in regulating the circulating BCAA concentrations. We therefore hypothesized that first-pass metabolism in the gut reduces the entry of colostrum BCAA into the systemic circulation, which in turn might have nutritional and physiological significance. It has been shown that besides Gln and Asn, a considerable amount of BCAA is used and oxidized by the intestine (Zhang et al., 2017). Previous studies have shown a high capacity of BCAA catabolism (transamination of BCAA) via BCAT, but the oxidation of the BCAA-derived branched-chain $\alpha$-ketoacids by BCKD in the intestinal mucosal cells of neonatal pigs was reported to be limited (about $30 \%$; Chen et al., 2007, 2009). In the current study, the mRNA abundance of BCATm, BCKDHA, and BCK$D H B$ did not differ between the groups, suggesting that colostrum intake was associated with better development of the intestinal epithelia in colostrum-fed calves (Steinhoff-Wagner et al., 2014) and with stimulation of anabolic processes, including protein syntheses in the small intestine of the COL calves. However, measuring mRNA might not mirror protein expression and thus, further studies including protein abundance and activity of BCAT and BCKDH are warranted to support our assumption.

It is known that postnatal intestinal development in neonates depends on the supply of nutrients, growth factors, and hormones (Blum, 2006; Zabielski et al., 2008; Lei et al., 2013). A positive association between a high content of Leu in sow's milk and rapid intestinal development has been reported in neonatal pigs (Wu and Knabe 1994; Wu et al., 2000), suggesting that BCAA, in particular Leu, are potentially important in regulating gastrointestinal development in neonates. A body of evidence shows that Leu acts as a signaling 


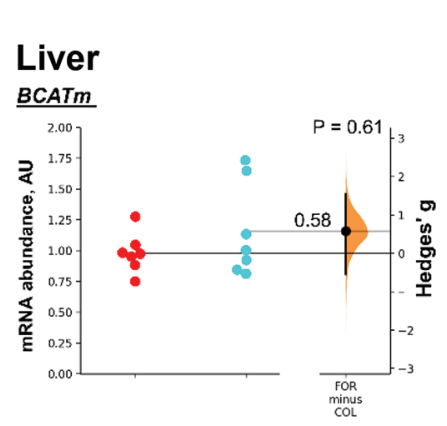

COL $\bigcirc$ FOR
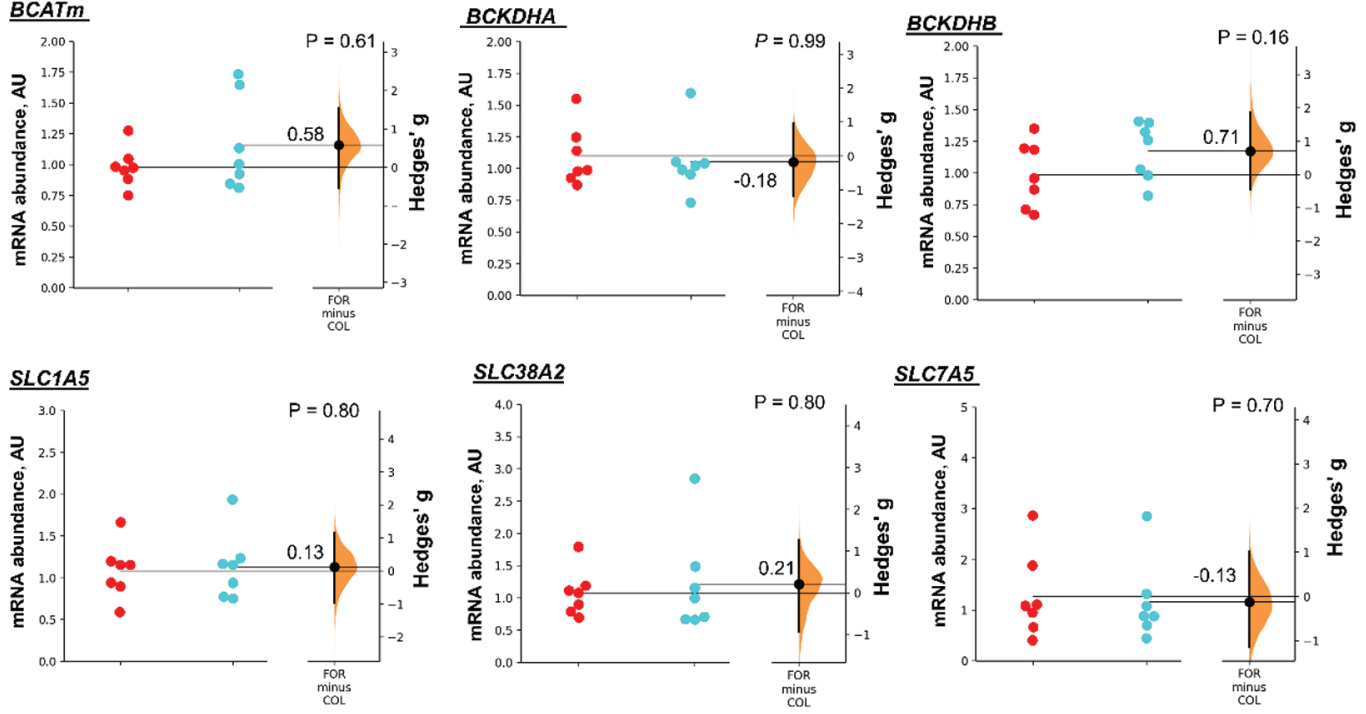

$\underline{\text { SLC7A5 }}$

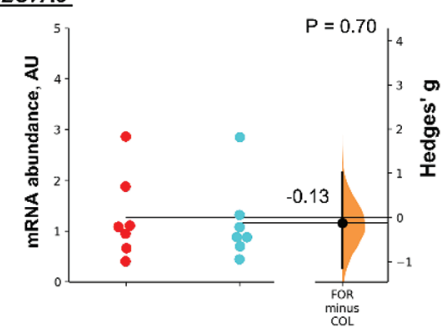

\section{Adipose tissue}
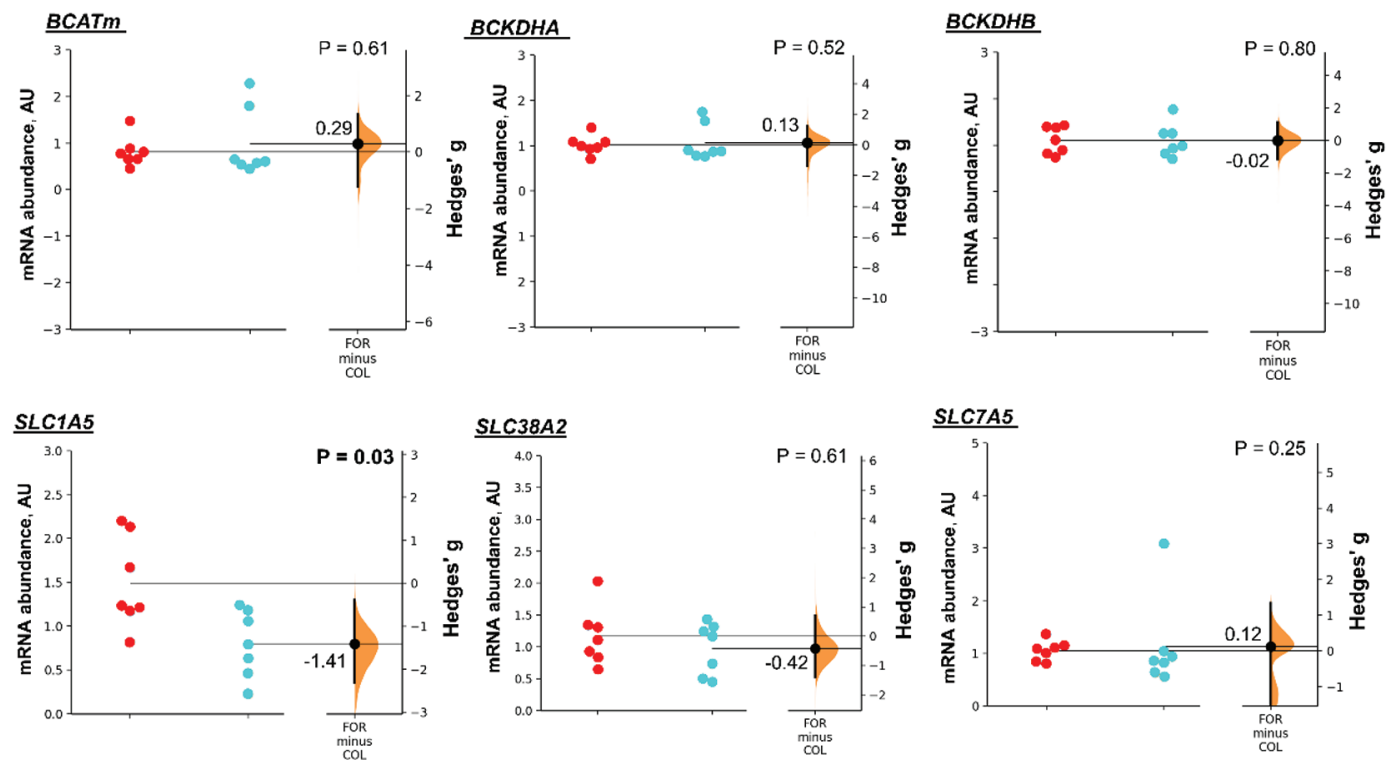

Figure 1. The mRNA abundance of genes related to branched-chain amino acid (BCAA) transporters and metabolizing enzymes in liver tissue and adipose tissue of calves fed either colostrum (COL) or formula (FOR) is shown as Gardner-Altman estimation plots. Both groups are plotted on the left axes; the mean difference is plotted on a floating axes on the right as a bootstrap sampling distribution. The mean difference is depicted as a dot; the $95 \%$ confidence interval is indicated by the ends of the vertical error bar. A Hedges' g of 1 shows that the 2 groups differ by 1 standard deviation. The $P$-values of the Mann-Whitney U-test for assessing the group effect is reported as the likelihood of observing the effect size.

molecule in the small intestine to activate the mammalian target of rapamycin, thereby stimulating protein synthesis and inhibiting proteolysis (Rhoads and $\mathrm{Wu}$, 2009), as well as enhancing the intestinal development in weaned piglets fed a low-protein diet (Zhang et al., 2013). On the other hand, several of the ingested colostral bioactive factors (such as insulin and IGF-I) have specific receptors localized in the mucosa of the small intestine, also demonstrated in calves (Hammon and Blum, 2002), and colon and can thus exert regulatory functions in neonates, including stimulating intestinal development (Blum, 2006; Hammon et al., 2012; Ontsouka et al., 2016). In view of the greater concentrations of free BCAA, IGF-I, and insulin in colostrum 
than in formula (Sadri et al., 2017), with the latter 2 plasma BCAA concentrations in the COL group (Saknown as critical factors mediating the stimulation of dri et al., 2017) might point to colostral BCAA being tissue protein synthesis in response to feeding (Davis favorably used for anabolic metabolism in the small et al., 2010), the observed decrease of the postprandial intestine of the neonatal calves, probably due to the

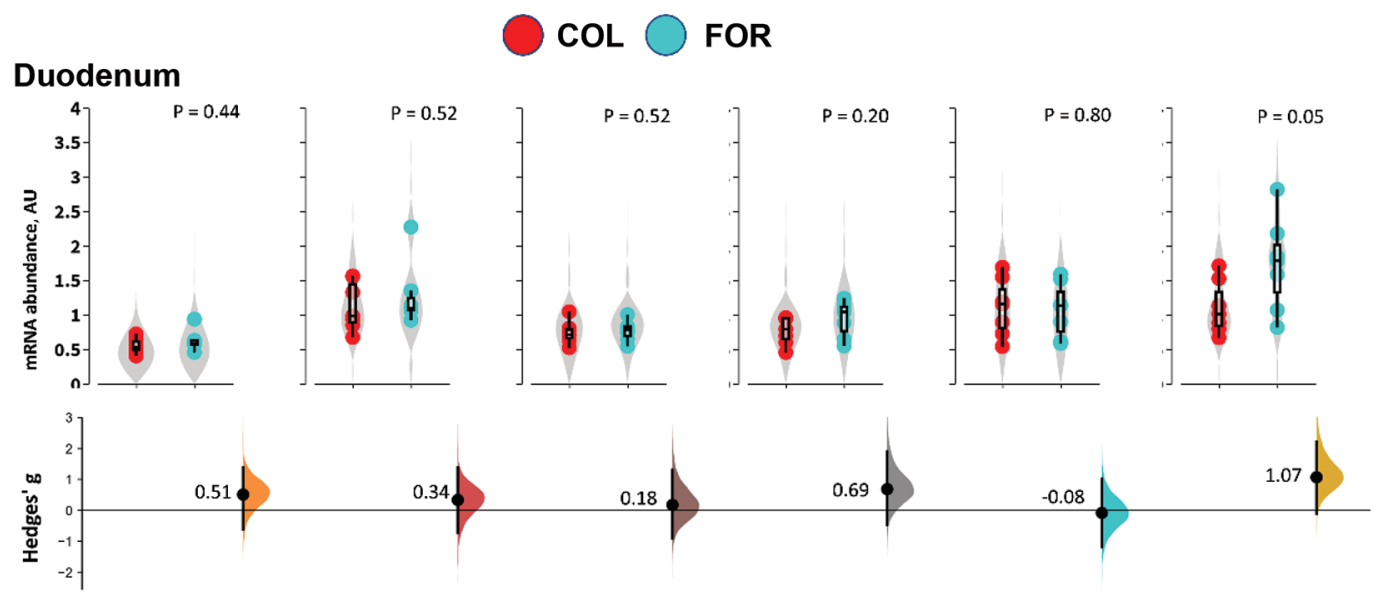

Jejunum (Proximal)

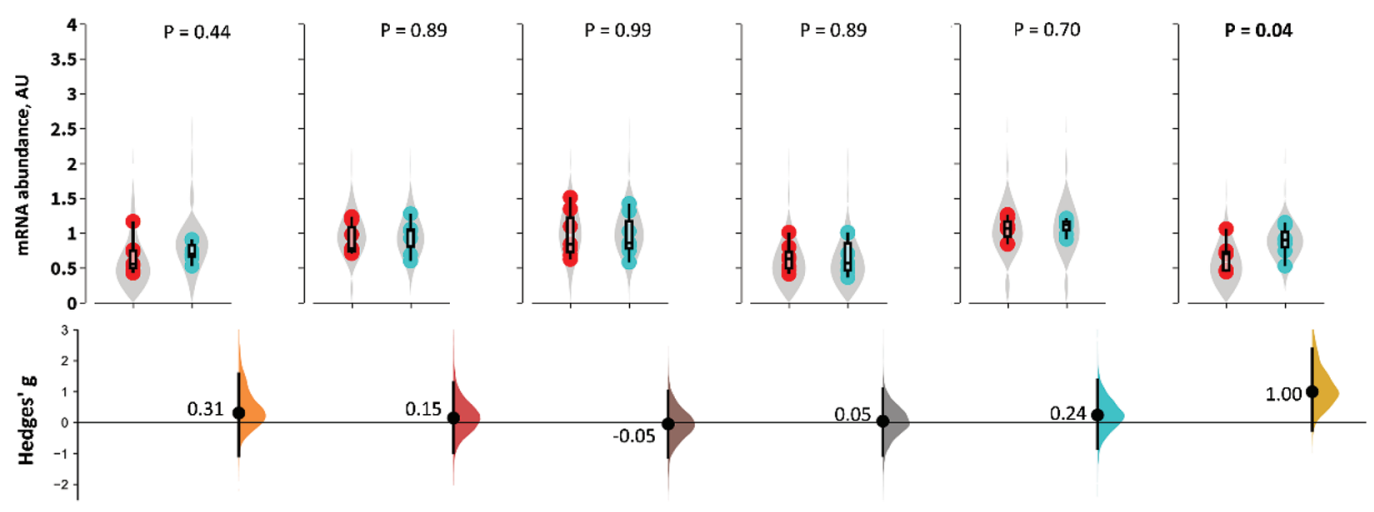

Jejunum (Middle)
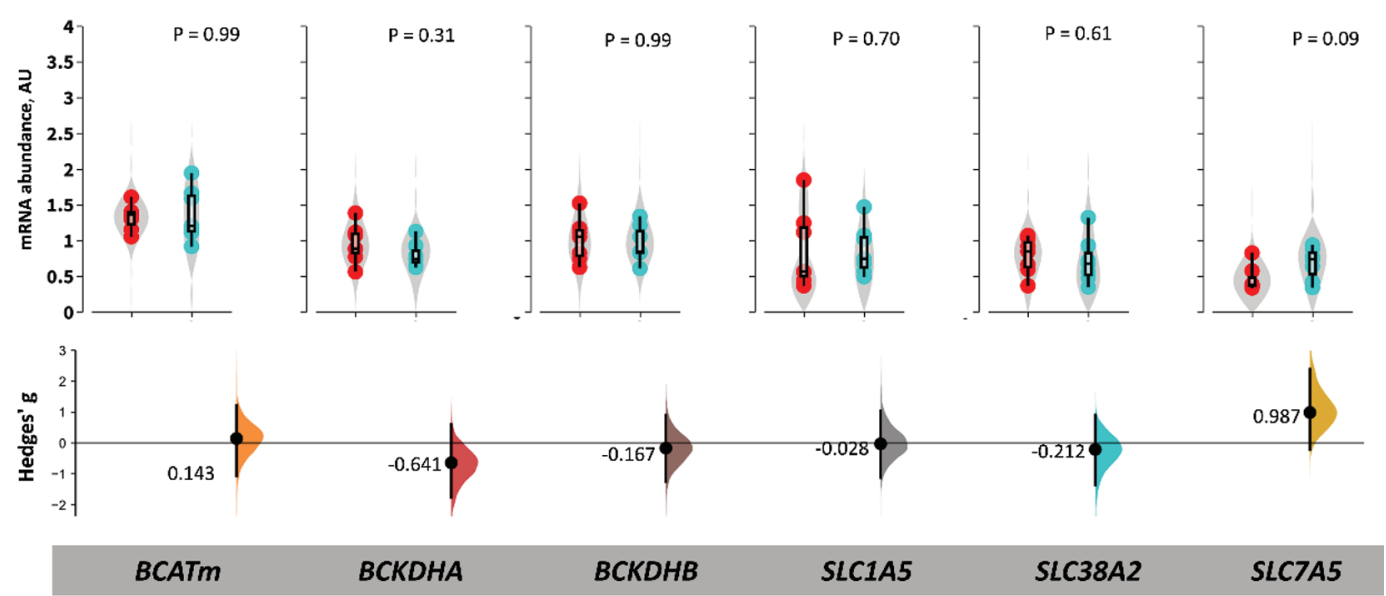

Figure 2. The mRNA abundance of genes related to branched-chain amino acid (BCAA) transporters and metabolizing enzymes in different small intestine sections. In each box, the horizontal line crossing the box is the median, the bottom and top of the box are the lower and upper quartiles, and the whiskers follow the Tukey boxplot definition. Different letters indicate differences $(P<0.05)$ between the different sections of the small intestine. Calves were fed either colostrum (COL) or formula (FOR). 
presence of more growth factors and hormones in the colostrum compared with the formula.

The transfer of AA across cell membranes in the lumen of the small intestine is a 2-stage transcellular process that takes place through a complex system of multiple amino acid transporters (Poncet and Taylor 2013; Taylor, 2014). The first stage, the import of amino acids from the lumen into intestinal epithelial cells, is carried out by apical transporters and the second stage, the export of amino acids from the cells to the extracellular space and ultimately to the bloodstream, is mediated by other transporters (a mixture of uniporters and antiporters) present in the basolateral membrane of gut cells including SLC7A5/LAT1 (Bröer and Bröer, 2017; Scalise et al., 2018). Among the transporters examined in the present study, the mRNA abundance of $S L C 7 A 5$ in the proximal jejunum, middle jejunum, as well as duodenum was reduced and those of SLC1A5 and SLC38A2 were not affected by colostrum feeding. The beneficial effects of bioactive substances in colostrum such as growth hormone (GH), IGF-I, and insulin on the morphological growth and functional maturation of the GI tract as well as on the metabolic, endocrine, and health status of neonatal calves have already been documented (Blattler et al., 2001; Blum, 2006; Ontsouka et al., 2016). Also, the distribution of receptors that mediate the effects of ligands of the somatotropic axis and the insulin system, namely growth hormore receptor, IGF-receptor, and insulin receptor across the GI tract of neonatal calves imply the importance of these hormonal systems in mediating the postnatal development and adaptation of neonatal calves (Baumrucker et al., 1994; Blum, 2006, Ontsouka et al., 2016). A potential anabolic stimulatory effect of colostrum might likely

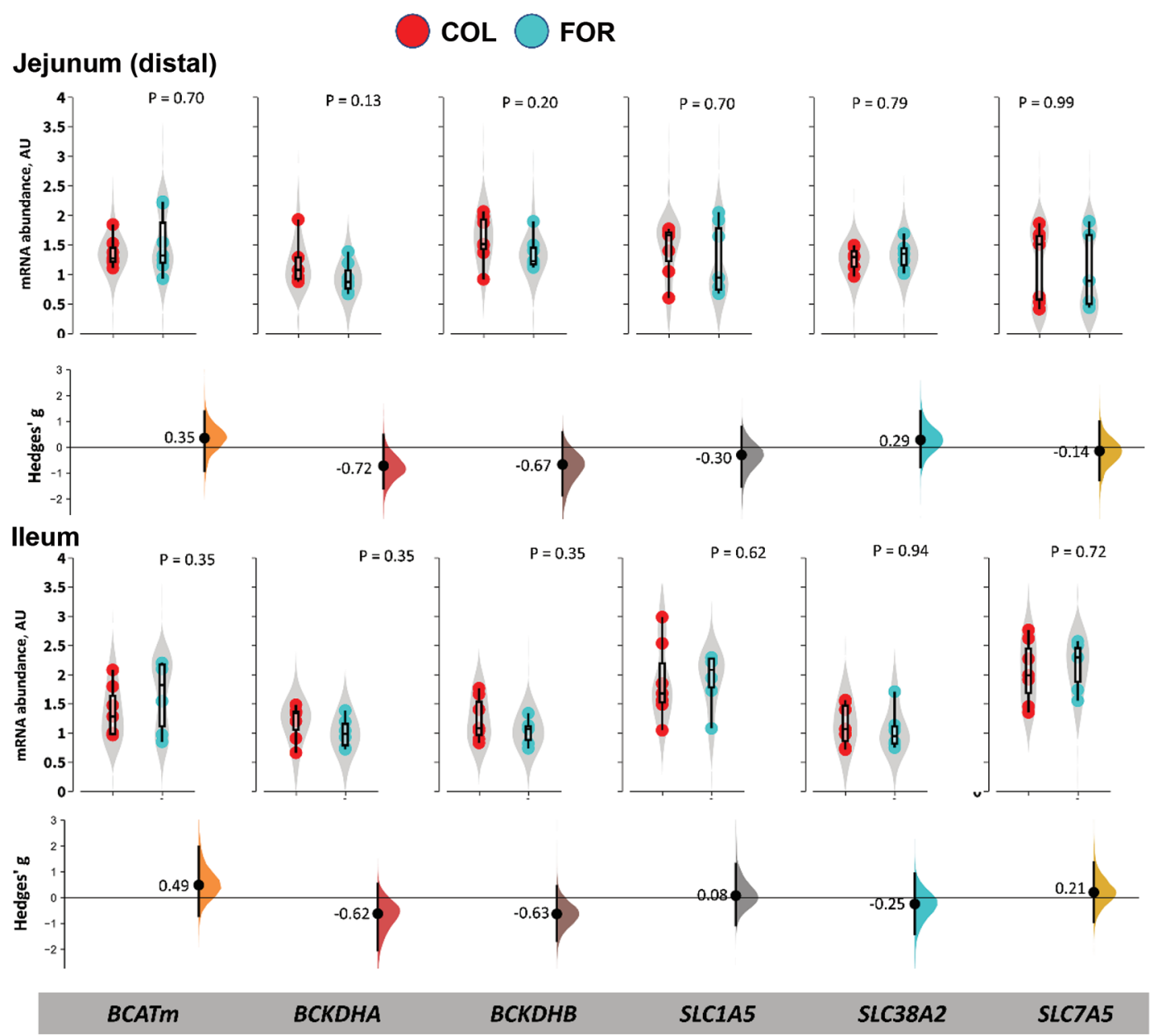

Figure 2 (Continued). The mRNA abundance of genes related to branched-chain amino acid (BCAA) transporters and metabolizing enzymes in different small intestine sections. In each box, the horizontal line crossing the box is the median, the bottom and top of the box are the lower and upper quartiles, and the whiskers follow the Tukey boxplot definition. Different letters indicate differences $(P<0.05)$ between the different sections of the small intestine. Calves were fed either colostrum (COL) or formula (FOR). 


\section{M. longissimus dorsi}

COL FOR

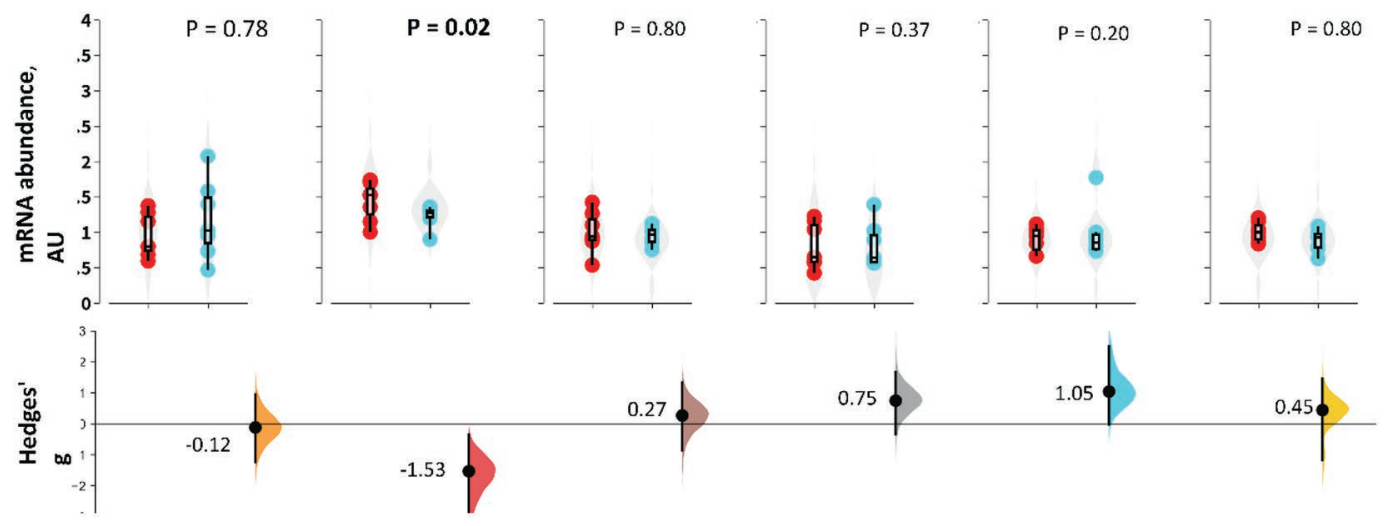

\section{M. masseter}
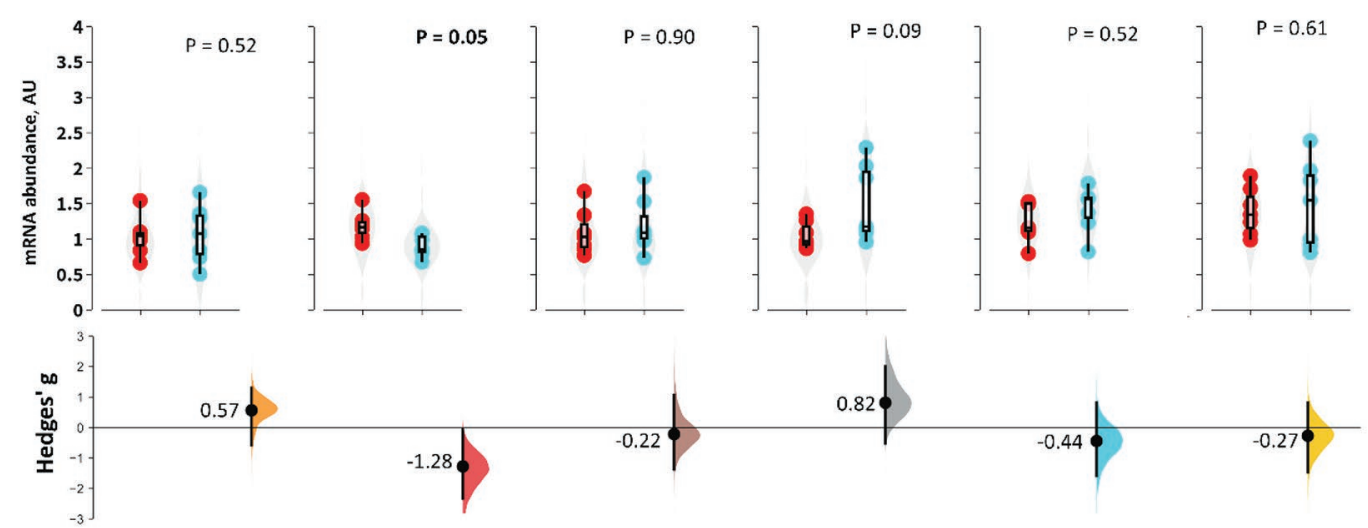

\section{M. semitendinosus}
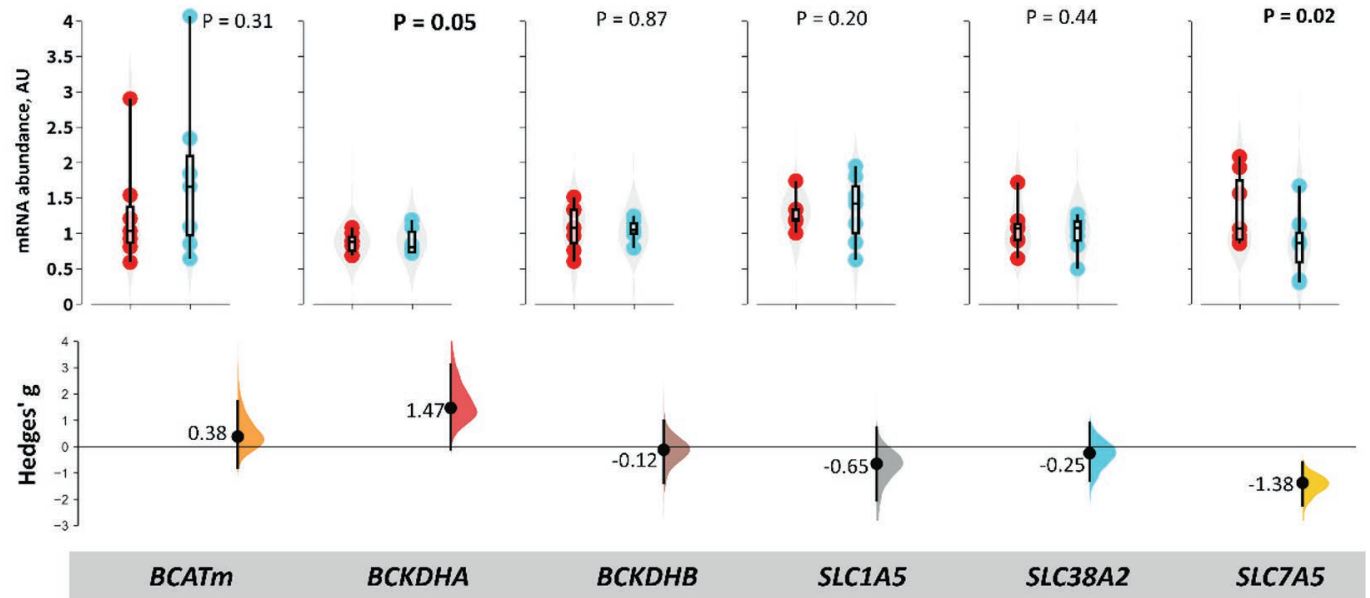

Figure 3. The mRNA abundance of genes related to branched-chain amino acid (BCAA) transporters and metabolizing enzymes in 3 different muscles of calves fed colostrum (COL) or formula (FOR) are shown as Cumming estimation plots. The raw data are plotted on the upper axes; each mean difference is plotted on the lower axes as a bootstrap sampling distribution. Mean differences are depicted as dots; $95 \%$ confidence intervals are indicated in the graph. A Hedges' g of 1 shows that the 2 groups differ by 1 standard deviation. The $P$-values of the Mann-Whitney U-test for assessing the group effect is reported as the likelihood of observing the effect size. 
be due to GH-IGF and insulin that are stimulated by increased BCAA levels in the small intestine.

It has been shown that the initial step in BCAA catabolism, the reversible deamination via BCAT, mainly takes place extrahepatically, such as in the muscle (Bequette et al., 2002), and in the adipose tissue (Bergen et al., 1988). Depending on the tissue, this is followed by either the export of the resulting BCKA into the bloodstream or direct oxidative decarboxylation of BCKA to acyl CoA derivatives via $\mathrm{BCKDH}$ and, consequently, not adding to the plasma flux (Lapierre et al., 2002). The differential effect of feeding colostrum on the mRNA abundance of $B C K D H A$ in the muscle might indicate a muscle type-specific response in the present study: In colostrum-fed calves, the mRNA abundance of $B C K D H A$ was increased in the MLD and MM whereas it was reduced in the MST. Such a muscle type-specific response to feeding colostrum was not accompanied by changes in the abundance of $B C K D H A$ mRNA in the liver and adipose tissue. Because of the lack or very low concentrations of bioactive substances in the formula, the utilization of BCAA in the intestinal cells of the formula-fed calves is probably less (or not) influenced by colostrum-borne insulin and IGF-I; as a consequence, the intestinal abundance of $S L C 7 A 5$ mRNA in the FOR calves was elevated to allow for greater export of BCAA from the intestinal cells to the bloodstream. This coincided with a lower abundance of $B C K D H A$ mRNA in the muscle, a rate-limiting factor in the catabolism of BCAA. These are potential contributing factors likely explaining the greater postprandial plasma BCAA concentrations in the FOR calves.

The greater mRNA abundance of $S L C 7 A 5$ in the MST of colostrum-fed calves coincided, as reported previously from this experiment (Sadri et al., 2017), with the upregulation of muscle ring finger protein-1 (MuRF-1; known as one of the major muscle-specific E3 ubiquitin ligases to control the ubiquitination and degradation of target proteins) and key components of mammalian target of rapamycin pathway (the major regulator of protein synthesis) at the level of the mRNA. These data might reflect a contribution of the $S L C 7 A 5$ in the higher rates of protein turnover in the COL calves through enhancing muscle neutral AA flux including BCAA.

Besides its major role in glucose and lipid homeostasis, adipose tissue also plays an important role in maintaining the balance of BCAA (Nie et al., 2018). A previous study in humans has demonstrated the potential capacity for adipose tissue to regulate circulating $\mathrm{BCAA}$ in vivo through the coordinated regulation of BCAA metabolizing enzymes in adipose tissue (Herman et al., 2010). Furthermore, Green et al. (2016) have reported that the catabolism of BCAA accounts for one-third of lipogenic acetyl-CoA generation and that the functional knockdown of BCAA degradation might impair lipid accumulation and adipocyte differentiation. In the current study, an increased mRNA abundance of SLC1A5 was observed in the adipose tissue of the COL calves as compared with the FOR calves. The SLC1A5/ ASCT2 mediates BCAA transport through net Gln uptake both directly and indirectly in a $\mathrm{Na}^{+}$dependent manner (Hundal and Taylor, 2009). The cellular uptake of Gln through SLC1A5 is considered as a rate-limiting step for the optimal intracellular concentration of EAA including BCAA and the activation of mammalian target of rapamycin C1 (Scalise et al., 2018). Through increased BCAA availability in the adipose tissue of the COL calves, they might fuel anaplerosis as well as de novo lipogenesis, though this assumption was not supported by concordant COL-related changes in BCAA catabolic enzymes mRNA in the adipose tissue.

In conclusion, the bioactive substances in colostrum are probably regulating the utilization of the BCAA in the intestinal cells through anabolic means that warrant further investigations. The differential effect of feeding colostrum on the abundance of $B C K D H A$ mRNA in different muscles might point to a muscle type-specific response. The unchanged hepatic mRNA abundance of BCAA transporters and catabolizing enzymes might imply a lack of involvement in BCAA metabolism in the first few d after birth in neonatal calves. Alternatively, it might indicate that the post-transcriptional modification of these factors comes into play and might have concealed their involvement.

\section{ACKNOWLEDGMENTS}

The authors thank J. Aschenbrenner from Bergophor Futtermittelfabrik GmbH (Kulmbach, Germany) for the donation of the formula and A. Zeyner (Martin Luther University Halle-Wittenberg, Halle and Wittenberg, Germany) for providing the animal facility. The authors also express their appreciation to Isabella Israel and Inga Hofs (Institute of Animal Science, Physiology and Hygiene Unit, University of Bonn, Bonn, Germany) for their excellent laboratory assistance. The financial support by Deutsche Forschungsgemeinschaft (Bonn, Germany) is also gratefully acknowledged (HA $4372 / 5-1)$. The authors have not stated any conflicts of interest.

\section{REFERENCES}

Ananieva, E. A., C. G. van Horn, M. R. Jones, and S. M. Hutson. 2017. Liver BCATm transgenic mouse model reveals the important role of the liver in maintaining BCAA homeostasis. J. Nutr. Biochem. 40:132-140. https://doi.org/10.1016/j.jnutbio.2016.10.014. 
Batistel, F., A. S. M. Alharthi, L. Wang, C. Parys, Y.-X. Pan, F. C. Cardoso, and J. J. Loor. 2017. Placentome nutrient transporters and mammalian target of rapamycin signaling proteins are altered by the methionine supply during late gestation in dairy cows and are associated with newborn birth weight. J. Nutr. 147:1640-1647. https://doi.org/10.3945/jn.117.251876.

Baumrucker, C. R., D. L. Hadsell, and J. W. Blum. 1994. Effects of dietary insulin-like growth factor I on growth and insulin-like growth factor receptors in neonatal calf intestine. J. Anim. Sci. 72:428-433. https://doi.org/10.2527/1994.722428x.

Bequette, B. J., C. E. Kyle, L. A. Crompton, S. E. Anderson, and M. D. Hanigan. 2002. Protein metabolism in lactating goats subjected to the insulin clamp. J. Dairy Sci. 85:1546-1555. https://doi.org/ 10.3168/jds.S0022-0302(02)74224-0.

Bergen, W. G., J. R. Busboom, and R. A. Merkel. 1988. Leucine degradation in sheep. Br. J. Nutr. 59:323-333. https://doi.org/10 $.1079 /$ BJN19880039.

Bionaz, M., and J. J. Loor. 2011. Gene networks driving bovine mammary protein synthesis during the lactation cycle. Bioinform. Biol. Insights 5:83-98. https://doi.org/10.4137/BBI.S7003.

Blattler, U., H. M. Hammon, C. Morel, C. Philipona, A. Rauprich, V. Rome, I. Le Huerou-Luron, P. Guilloteau, and J. W. Blum. 2001. Feeding colostrum, its composition and feeding duration variably modify proliferation and morphology of the intestine and digestive enzyme activities of neonatal calves. J. Nutr. 131:1256-1263.

Blum, J. W. 2006. Nutritional physiology of neonatal calves. J. Anim. Physiol. Anim. Nutr. (Berl.) 90:1-11. https://doi.org/10.1111/j .1439-0396.2005.00614.x.

Blum, J. W., and H. Hammon. 2000. Colostrum effects on the gastrointestinal tract, and on nutritional, endocrine, and metabolic parameters in neonatal calves. Livest. Prod. Sci. 66:151-159. https: //doi.org/10.1016/S0301-6226(00)00222-0.

Bröer, S., and A. Bröer. 2017. Amino acid homeostasis and signalling in mammalian cells and organisms. Biochem. J. 474:1935-1963. https://doi.org/10.1042/BCJ20160822.

Bühler, C., H. Hammon, G. L. Rossi, and J. W. Blum. 1998. Small intestinal morphology in eight-day-old calves fed colostrum for different durations or only milk replacer and treated with long- $\mathrm{R}^{3}$ insulin-like growth factor I and growth hormone. J. Anim. Sci. 76:758-765. https://doi.org/10.2527/1998.763758x.

Bustin, S. A., V. Benes, J. A. Garson, J. Hellemans, J. Huggett, M. Kubista, R. Mueller, T. Nolan, M. W. Pfaffl, G. L. Shipley, J. Vandesompele, and C. T. Wittwer. 2009. The MIQE guidelines: Minimum information for publication of quantitative real-time PCR experiments. Clin. Chem. 55:611-622. https://doi.org/10 .1373/clinchem.2008.112797.

Chen, L., P. Li, J. Wang, X. Li, H. Gao, Y. Yin, Y. Hou, and G. Wu. 2009. Catabolism of nutritionally essential amino acids in developing porcine enterocytes. Amino Acids 37:143-152. https://doi.org/ 10.1007/s00726-009-0268-1.

Chen, L., Y. L. Yin, W. S. Jobgen, S. C. Jobgen, D. A. Knabe, W. X. $\mathrm{Hu}$, and G. Wu. 2007. In vitro oxidation of essential amino acids by jejunal mucosal cells of growing pigs. Livest. Sci. 109:19-23. https://doi.org/10.1016/j.livsci.2007.01.027.

Claridge-Chang, A., and P. N. Assam. 2016. Estimation statistics should replace significance testing. Nat. Methods 13:108-109. https://doi.org/10.1038/nmeth.3729.

Crichton, N. 2000. Information point: Mann-Whitney test. J. Clin. Nurs. 9:583

David, J., D. Dardevet, L. Mosoni, I. Savary-Auzeloux, and S. Polakof. 2019. Impaired skeletal muscle branched-chain amino acids catabolism contributes to their increased circulating levels in a nonobese insulin-resistant fructose-fed rat model. Nutrients 11:355. https://doi.org/10.3390/nu11020355.

Davis, T. A., A. Suryawan, R. A. Orellana, M. L. Fiorotto, and D. G. Burrin. 2010. Amino acids and insulin are regulators of muscle protein synthesis in neonatal pigs. Animal 4:1790-1796. https:// doi.org/10.1017/S1751731110000984.

de Groof, F., L. S. Huang, I. van Vliet, G. J. Voortman, H. Schierbeek, L. C. W. Roksnoer, A. Vermes, C. Chen, Y. Huang, and J. B. van Goudoever. 2014. Branched-chain amino acid requirements for enterally fed term neonates in the first month of life. Am. J. Clin. Nutr. 99:62-70. https://doi.org/10.3945/ajcn.112.038927.

Ghaffari, M. H., A. Jahanbekam, H. Sadri, K. Schuh, G. Dusel, C. Prehn, J. Adamski, C. Koch, and H. Sauerwein. 2019. Metabolomics meets machine learning: Longitudinal metabolite profiling in serum of normal versus overconditioned cows and pathway analysis. J. Dairy Sci. 102:11561-11585. https://doi.org/10.3168/ jds.2019-17114.

Green, C. R., M. Wallace, A. S. Divakaruni, S. A. Phillips, A. N. Murphy, T. P. Ciaraldi, and C. M. Metallo. 2016. Branchedchain amino acid catabolism fuels adipocyte differentiation and lipogenesis. Nat. Chem. Biol. 12:15-21. https://doi.org/10.1038/ nchembio.1961.

Greenland, S., S. J. Senn, K. J. Rothman, J. B. Carlin, C. Poole, S. N. Goodman, and D. G. Altman. 2016. Statistical tests, $P$ values, confidence intervals, and power: A guide to misinterpretations. Eur. J. Epidemiol. 31:337-350. https://doi.org/10.1007/s10654 $-016-0149-3$.

Gruse, J., S. Görs, A. Tuchscherer, W. Otten, J. M. Weitzel, C. C. Metges, S. Wolffram, and H. M. Hammon. 2015. The effects of oral quercetin supplementation on splanchnic glucose metabolism in 1-week-old calves depend on diet after birth. J. Nutr. 145:24862495. https://doi.org/10.3945/jn.115.218271.

Hammon, H., and J. W. Blum. 1999. Free amino acids in plasma of neonatal calves are influenced by feeding colostrum for different durations or by feeding only milk replacer. J. Anim. Physiol. Anim. Nutr. (Berl.) 82:193-204. https://doi.org/10.1046/j.1439 $-0396.1999 .00229 . x$.

Hammon, H. M., and J. W. Blum. 2002. Feeding different amounts of colostrum or only milk replacer modify receptors of intestinal insulin-like growth factors and insulin in neonatal calves. Domest. Anim. Endocrinol. 22:155-168. https://doi.org/10.1016/S0739 $-7240(02) 00122-4$.

Hammon, H. M., J. Steinhoff-Wagner, U. Schönhusen, C. C. Metges, and J. W. Blum. 2012. Energy metabolism in the newborn farm animal with emphasis on the calf: Endocrine changes and responses to milk-born and systemic hormones. Domest. Anim. Endocrinol. 43:171-185. https://doi.org/10.1016/j.domaniend.2012.02.005.

Harper, A. E., R. Miller, and K. P. Block. 1984. Branched-chain amino acid metabolism. Annu. Rev. Nutr. 4:409-454. https://doi.org/10 .1146/annurev.nu.04.070184.002205.

Hellemans, J., G. Mortier, A. De Paepe, F. Speleman, and J. Vandesompele. 2007. qBase relative quantification framework and software for management and automated analysis of real-time quantitative PCR data. Genome Biol. 8:R19. https://doi.org/10.1186/ gb-2007-8-2-r19.

Herman, M. A., P. She, O. D. Peroni, C. J. Lynch, and B. B. Kahn. 2010. Adipose tissue branched chain amino acid (BCAA) metabolism modulates circulating BCAA levels. J. Biol. Chem. 285:11348-11356. https://doi.org/10.1074/jbc.M109.075184.

Hernández-Castellano, L. E., A. Arguello, A. M. Almeida, N. Castro, and E. Bendixen. 2015. Colostrum protein uptake in neonatal lambs examined by descriptive and quantitative liquid chromatography-tandem mass spectrometry. J. Dairy Sci. 98:135-147. https: //doi.org/10.3168/jds.2014-8143.

Hundal, H. S., and P. M. Taylor. 2009. Amino acid transporters: Gate keepers of nutrient exchange and regulators of nutrient signaling. Am. J. Physiol. Endocrinol. Metab. 296:E603-E613. https://doi .org/10.1152/ajpendo.91002.2008.

Hyde, R., P. M. Taylor, and H. S. Hundal. 2003. Amino acid transporters: Roles in amino acid sensing and signalling in animal cells. Biochem. J. 373:1-18. https://doi.org/10.1042/bj20030405.

Kandasamy, P., G. Gyimesi, Y. Kanai, and M. A. Hediger. 2018. Amino acid transporters revisited: New views in health and disease. Trends Biochem. Sci. 43:752-789. https://doi.org/10.1016/j.tibs .2018.05.003.

Kargar, S., M. Roshan, S. M. Ghoreishi, A. Akhlaghi, M. Kanani, A. R. Abedi Shams-Abadi, and M. H. Ghaffari. 2020. Extended colostrum feeding for two weeks improves growth performance and reduces the susceptibility to diarrhea and pneumonia in neonatal 
Holstein dairy calves. J. Dairy Sci. 103:8130-8142. https://doi. org/10.3168/jds.2020-18355.

Kashyap, S., and C. W. Heird. 2011. Protein and amino acid metabolism and requirements. Pages 603-612 in Fetal and Neonatal Physiology. 4th ed. Elsevier/Saunders, Philadelphia, PA.

Lapierre, H., J. P. Blouin, J. F. Bernier, C. K. Reynolds, P. Dubreuil, and G. E. Lobley. 2002. Effect of supply of metabolizable protein on whole body and splanchnic leucine metabolism in lactating dairy cows. J. Dairy Sci. 85:2631-2641. https://doi.org/10.3168/ jds.S0022-0302(02)74348-8.

Lei, J., D. Y. Feng, Y. L. Zhang, S. Dahanayaka, X. L. Li, K. Yao, J. J. Wang, Z. L. Wu, Z. L. Dai, and G. Y. Wu. 2013. Hormonal regulation of leucine catabolism in mammary epithelial cells. Amino Acids 45:531-541. https://doi.org/10.1007/s00726-012-1332-9.

Liang, Y., F. Batistel, C. Parys, and J. J. Loor. 2019. Methionine supply during the periparturient period enhances insulin signaling, amino acid transporters, and mechanistic target of rapamycin pathway proteins in adipose tissue of Holstein cows. J. Dairy Sci. 102:4403-4414. https://doi.org/10.3168/jds.2018-15738.

Ma, T., E. O'Hara, Y. Song, A. J. Fischer, Z. He, M. A. Steele, and L. L. Guan. 2019. Altered mucosa-associated microbiota in the ileum and colon of neonatal calves in response to delayed first colostrum feeding. J. Dairy Sci. 102:7073-7086. https://doi.org/10.3168/jds 2018-16130.

Malmuthuge, N., Y. Chen, G. Liang, L. A. Goonewardene, and L. L. Guan. 2015. Heat-treated colostrum feeding promotes beneficial bacteria colonization in the small intestine of neonatal calves. J. Dairy Sci. 98:8044-8053. https://doi.org/10.3168/jds.2015-9607.

Naumann, C., and R. Bassler. 2012. Die chemische Untersuchung von Futtermitteln. VDLUFA-Verlag, Darmstadt, Germany.

Neinast, M., D. Murashige, and Z. Arany. 2019. Branched chain amino acids. Annu. Rev. Physiol. 81:139-164. https://doi.org/10.1146/ annurev-physiol-020518-114455.

Nie, C., T. He, W. Zhang, G. Zhang, and X. Ma. 2018. Branched chain amino acids: Beyond nutrition metabolism. Int. J. Mol. Sci. 19:954. https://doi.org/10.3390/ijms19040954.

Ontsouka, E. C., C. Albrecht, and R. M. Bruckmaier. 2016. Invited review: Growth-promoting effects of colostrum in calves based on interaction with intestinal cell surface receptors and receptor-like transporters. J. Dairy Sci. 99:4111-4123. https://doi.org/10.3168/ jds.2015-9741.

Poncet, N., and P. M. Taylor. 2013. The role of amino acid transporters in nutrition. Curr. Opin. Clin. Nutr. Metab. Care 16:57-65. https://doi.org/10.1097/MCO.0b013e32835a885c.

Rhoads, J. M., and G. Wu. 2009. Glutamine, arginine, and leucine signaling in the intestine. Amino Acids 37:111-122. https://doi.org/ $10.1007 / \mathrm{s} 00726-008-0225-4$.

Sadri, H., M. H. Ghaffari, K. Schuh, G. Dusel, C. Koch, C. Prehn, J. Adamski, and H. Sauerwein. 2020. Metabolome profiling in skeletal muscle to characterize metabolic alterations of overconditioned cows during the periparturient period. J. Dairy Sci. 103:3730-3744. https://doi.org/10.3168/jds.2019-17566.

Sadri, H., J. Steinhoff-Wagner, H. M. Hammon, R. M. Bruckmaier, S. Görs, and H. Sauerwein. 2017. Mammalian target of rapamycin signaling and ubiquitin proteasome-related gene expression in 3 different skeletal muscles of colostrum- versus formula-fed calves. J. Dairy Sci. 100:9428-9441. https://doi.org/10.3168/jds.2017 -12857 .

Scalise, M., M. Galluccio, L. Console, L. Pochini, and C. Indiveri. 2018. The human SLC7A5 (LAT1): The intriguing histidine/large neutral amino acid transporter and its relevance to human health. Front. Chem. 6:243.

Steinhoff-Wagner, J., S. Görs, P. Junghans, R. M. Bruckmaier, E. Kanitz, C. C. Metges, and H. M. Hammon. 2011. Intestinal glucose absorption but not endogenous glucose production differs between colostrum- and formula-fed neonatal calves. J. Nutr. 141:48-55. https://doi.org/10.3945/jn.110.128652.

Steinhoff-Wagner, J., R. Zitnan, U. Schönhusen, H. Pfannkuche, M. Hudakova, C. C. Metges, and H. M. Hammon. 2014. Diet effects on glucose absorption in the small intestine of neonatal calves: Importance of intestinal mucosal growth, lactase activity, and glucose transporters. J. Dairy Sci. 97:6358-6369. https://doi.org/10.3168/ jds.2014-8391.

Suryawan, A., and T. A. Davis. 2011. Regulation of protein synthesis by amino acids in muscle of neonates. Front Biosci (Landmark Ed) 16:1445-1460.

Taylor, P. M. 2014. Role of amino acid transporters in amino acid sensing. Am. J. Clin. Nutr. 99:223S-230S. https://doi.org/10.3945/ ajcn.113.070086.

Webb, L. A., H. Sadri, K. Schuh, S. Egert, P. Stehle, I. Meyer, C. Koch, G. Dusel, and H. Sauerwein. 2020. Branched-chain amino acids: Abundance of their transporters and metabolizing enzymes in adipose tissue, skeletal muscle, and liver of dairy cows at high or normal body condition. J. Dairy Sci. 103:2847-2863. https://doi .org/10.3168/jds.2019-17147.

Webb, L. A., H. Sadri, D. von Soosten, S. Dänicke, S. Egert, P. Stehle, and H. Sauerwein. 2019. Changes in tissue abundance and activity of enzymes related to branched-chain amino acid catabolism in dairy cows during early lactation. J. Dairy Sci. 102:3556-3568. https://doi.org/10.3168/jds.2018-14463.

Wu, G., N. E. Flynn, and D. A. Knabe. 2000. Enhanced intestinal synthesis of polyamines from proline in cortisol-treated piglets. Am. J. Physiol. Endocrinol. Metab. 279:E395-E402. https://doi.org/10 .1152/ajpendo.2000.279.2.E395.

Wu, G., and D. A. Knabe. 1994. Free and protein-bound amino-acids in sow's colostrum and milk. J. Nutr. 124:415-424. https://doi .org/10.1093/jn/124.3.415.

Zabielski, R., M. M. Godlewski, and P. Guilloteau. 2008. Control of development of gastrointestinal system in neonates. J. Physiol. Pharmacol. 59:35-54.

Zhang, S., S. Qiao, M. Ren, X. Zeng, X. Ma, Z. Wu, P. Thacker, and G. Wu. 2013. Supplementation with branched-chain amino acids to a low-protein diet regulates intestinal expression of amino acid and peptide transporters in weanling pigs. Amino Acids 45:11911205. https://doi.org/10.1007/s00726-013-1577-y.

Zhang, S., X. Zeng, M. Ren, X. Mao, and S. Qiao. 2017. Novel metabolic and physiological functions of branched-chain amino acids: A review. J. Anim. Sci. Biotechnol. 8:10. https://doi.org/10.1186/ s40104-016-0139-z. 